\title{
Original
}

\section{Effects of miglitol, sitagliptin or their combination on plasma glucose, insulin and incretin levels in non-diabetic men}

\author{
Kazutaka Aoki ${ }^{1,2)}$, Kiyomi Masuda ${ }^{2)}$, Takashi Miyazaki ${ }^{2)}$, Yu Togashi ${ }^{2)}$ and Yasuo Terauchi²) \\ ${ }^{1)}$ Yokosuka Kyousai Hospital, Yokosuka 238-8558, Japan \\ ${ }^{2)}$ Department of Endocrinology and Metabolism, Yokohama City University Graduate School of Medicine, Yokohama 236-0004, Japan
}

\begin{abstract}
GIs) increase active glucagon-like peptide-1 (GLP-1) and reduce the total glucosedependent insulinotropic polypeptide (GIP) levels, but their ability to prevent diabetes remains uncertain. Dipeptidyl peptidase-4 (DPP-4) inhibitors, such as sitagliptin, increase active GLP-1 and GIP levels and improve hyperglycemia in a glucose-dependent fashion. However, the effectiveness of their combination in subjects with normal glucose tolerance (NGT) or impaired glucose tolerance (IGT) is uncertain. The present study evaluated the effect of miglitol, sitagliptin, and their combination on glucose, insulin and incretin levels in non-diabetic men. Miglitol and sitagliptin were administered according to four different intake schedules (C: no drug, M: miglitol; S: sitagliptin, M+S: miglitol and sitagliptin). The plasma glucose levels were significantly lower for $\mathrm{M}, \mathrm{S}$ and $\mathrm{M}+\mathrm{S}$ than for the control. The areas under the curve (AUCs) of the plasma active GLP-1 level in the $\mathrm{M}, \mathrm{S}$, and $\mathrm{M}+\mathrm{S}$ groups were significantly greater than that in the control group. The AUC of the plasma active GLP-1 level was significantly greater for $\mathrm{M}+\mathrm{S}$ group than for the $\mathrm{M}$ and $\mathrm{S}$ groups. The AUC of the plasma total GIP level was significantly smaller for $\mathrm{M}+\mathrm{S}$ group than for the control and $\mathrm{M}$ and $\mathrm{S}$ groups. The results of our study suggest that miglitol, sitagliptin, or their combination contributes to the prevention of type 2 diabetes.
\end{abstract}

Key words: Miglitol, $\alpha$-glucosidase inhibitor, Sitagliptin, DPP-4 inhibitor, Postprandial hyperglycemia

$\boldsymbol{\alpha}$-GLUCOSIDASE inhibitors $(\alpha \mathrm{GIs})$ decrease plasma glucose and serum insulin levels in healthy subjects $[1,2]$ and reduce the development of type 2 diabetes in subjects with impaired glucose tolerance (IGT) $[3,4]$. aGIs reportedly enhance active glucagon-like peptide-1 (GLP-1) responses and reduce total glucose-dependent insulinotropic polypeptide (GIP) responses [5-7]; however, their significance for protecting against the development of diabetes remains uncertain. Dipeptidyl peptidase-4 (DPP-4) inhibitors, such as sitagliptin, increase active GLP-1 and GIP by inhibiting DPP-4 enzymatic activity and improve hyperglycemia in a glucose-dependent fashion by increasing serum insulin and decreasing serum glucagon levels in diabetic patients [8]. Since

Received Apr. 1, 2010; Accepted May 8, 2010 as K10E-103 Released online in J-STAGE as advance publication Jun. 1, 2010

Correspondence to: Yasuo Terauchi, Department of Endocrinology and Metabolism, Yokohama City University Graduate School of Medicine, Fuku-ura 3-9, Kanazawa-ku, Yokohama 236-0004, Japan.

E-mail: terauchi@yokohama-cu.ac.jp
GLP-1 reportedly promotes islet cell growth and inhibits apoptosis in animal models [9], an elevated plasma GLP-1 level might promote $\beta$ cell protection in addition to improving hyperglycemia in humans. Given the fact that diabetes develops when insulin secretion from $\beta$ cells is insufficient to compensate for insulin resistance [10,11], DPP-4 inhibitors may reduce the development of type 2 diabetes in subjects with IGT or normal glucose tolerance (NGT). It is reported that vildagliptin increases active GLP-1 level and decreases postprandial glucose and glucagon levels without changing insulin level in subjects with IGT [12]. It is also reported that sitagliptin increases active GLP-1without changing postprandial glucose, insulin or glucagon levels in subjects with NGT and impaired fasting glucose (IFG) [13, 14]. However, the effects of a combination of $\alpha$ GIs and DPP- 4 inhibitors on blood glucose, insulin and incretin levels in pre-diabetic subjects are uncertain. We therefore evaluated the effect of miglitol, sitagliptin, and their combination on these parameters in non-diabetic men. 


\section{Materials and Methods}

After obtaining approval from the Institutional Ethics Review Committee, 10 healthy men aged 37 \pm 2 years (with a BMI of $24.1 \pm 0.6 \mathrm{~kg} / \mathrm{m}^{2}$ ) who had never been diagnosed as having diabetes were enrolled in the present study. Informed consent was obtained from each of the subjects prior to the start of the study.

Miglitol and/or sitagliptin were administered according to four different intake schedules (C: no drug, M: miglitol administered just before a meal [50 mg]; S: sitagliptin administered at 2 hours before the start of a meal [50 mg], M+S: miglitol administered just before a meal [50 mg] and sitagliptin administered at 2 hours before the start of a meal [50 mg]). The subjects were randomized to one of the four interventions using a crossover design. Subjects were asked to take each medication after more than 1 week drug-free washout period. All the subjects received a standard breakfast (773 Kcal; protein: 27.0 grams; fat: 20.3 grams; carbohydrate: 121.5 grams). For the study, the subjects were requested to fast for at least 12 hours. Blood samples were collected at $0,30,60,120$ and $180 \mathrm{~min}$ after the start of breakfast. The plasma glucose and serum insulin levels were measured, and the plasma active GLP-1 and plasma total GIP levels were measured using ELISA kits (Millipore Corporation, MA, USA) at SRL, Inc. (Tokyo, Japan). We measured the total GIP in this study because we could not obtain commercially available kits for measuring active GIP accurately.

Data are expressed as the means $\pm \mathrm{SE}$. The areas under the curve (AUC) from just before a meal to 180 min after the start of a meal were calculated using the trapezoid method. The analyses were performed using a two-way layout analysis of variance (ANOVA) with Tukey-type multiple comparisons.

\section{Results}

The time profiles and AUCs of the plasma glucose and serum insulin levels are shown in Fig. 1. The plasma glucose levels at $30 \mathrm{~min}$ after the start of breakfast were significantly lower in the $\mathrm{M}$ and $\mathrm{M}+\mathrm{S}$ groups than in the control group, while the plasma glucose levels at $60 \mathrm{~min}$ after the start of breakfast were significantly lower in all the intake groups than in the C group (Fig. 1A). The AUCs of the plasma glucose levels in the $\mathrm{M}, \mathrm{S}$ and $\mathrm{M}+\mathrm{S}$ groups were significantly lower than that in the $\mathrm{C}$ group; however, no significant differences between the $\mathrm{M}+\mathrm{S}$ group and the $\mathrm{M}$ or $\mathrm{S}$ groups were observed (Fig. 1B).

The serum insulin levels at 30 and 60 min after the start of breakfast were significantly lower in the $\mathrm{M}$ and $\mathrm{M}+\mathrm{S}$ groups than in the control group (Fig. 1C). The AUCs of the serum insulin levels in the $\mathrm{M}$ and $\mathrm{M}+\mathrm{S}$ groups were significantly smaller than that in the control group (Fig. 1D). By contrast, the AUC of the serum insulin levels was unaffected in the $\mathrm{S}$ group.

The time profiles and the AUCs of the plasma active GLP-1 and plasma total GIP levels are shown in Fig. 2. The active GLP-1 levels at 0 min were significantly higher in the intake $\mathrm{S}$ and $\mathrm{M}+\mathrm{S}$ groups than in the $\mathrm{C}$ and $\mathrm{M}$ groups (Fig. 2A), probably because the sitagliptin was administered 2 hours before the meal. The active GLP-1 levels at 30, 120 and $180 \mathrm{~min}$ after the start of breakfast were significantly higher in the $\mathrm{S}$ and $\mathrm{M}+\mathrm{S}$ groups than in the $\mathrm{C}$ group, and the active GLP-1 levels at 60 min after the start of breakfast were significantly higher in all the intake groups than in the control group (Fig. 2A). The AUCs of the plasma active GLP-1 level in the M, S and $\mathrm{M}+\mathrm{S}$ groups were significantly greater than that in the control group. Thus, the AUC of the plasma active GLP-1 level increased by $38 \%$ in the $\mathrm{M}$ group, $78 \%$ in the $\mathrm{S}$ group, and $153 \%$ in the $\mathrm{M}+\mathrm{S}$ group, relative to the value in the control group. Furthermore, the AUC of the $\mathrm{M}+\mathrm{S}$ group was significantly greater than those of the $\mathrm{M}$ or $\mathrm{S}$ group (Fig. 2B).

The total GIP levels at 30 and 60 min after the start of breakfast were significantly lower in all the intake groups than in the $\mathrm{C}$ group, and the GIP levels at 120 and $180 \mathrm{~min}$ after the start of breakfast were significantly lower in the $\mathrm{M}+\mathrm{S}$ group than in the $\mathrm{C}$ and $\mathrm{M}$ groups (Fig. 2C). The AUCs of the plasma total GIP level in the $\mathrm{M}, \mathrm{S}$ and $\mathrm{M}+\mathrm{S}$ groups were significantly smaller than that in the $\mathrm{C}$ group. Thus, the AUC of the total GIP levels decreased by $28 \%$ in the M group, $34 \%$ in the $\mathrm{S}$ group, and $64 \%$ in the $\mathrm{M}+\mathrm{S}$ group, compared with the value in the $\mathrm{C}$ group. Furthermore, the AUC of the $\mathrm{M}+\mathrm{S}$ group was significantly smaller than those of the $\mathrm{M}$ or $\mathrm{S}$ groups (Fig. 2D).

\section{Discussion}

Here, we report two important findings: the plasma glucose levels in the $\mathrm{M}, \mathrm{S}$ and $\mathrm{M}+\mathrm{S}$ groups were significantly lower than those in the control group 

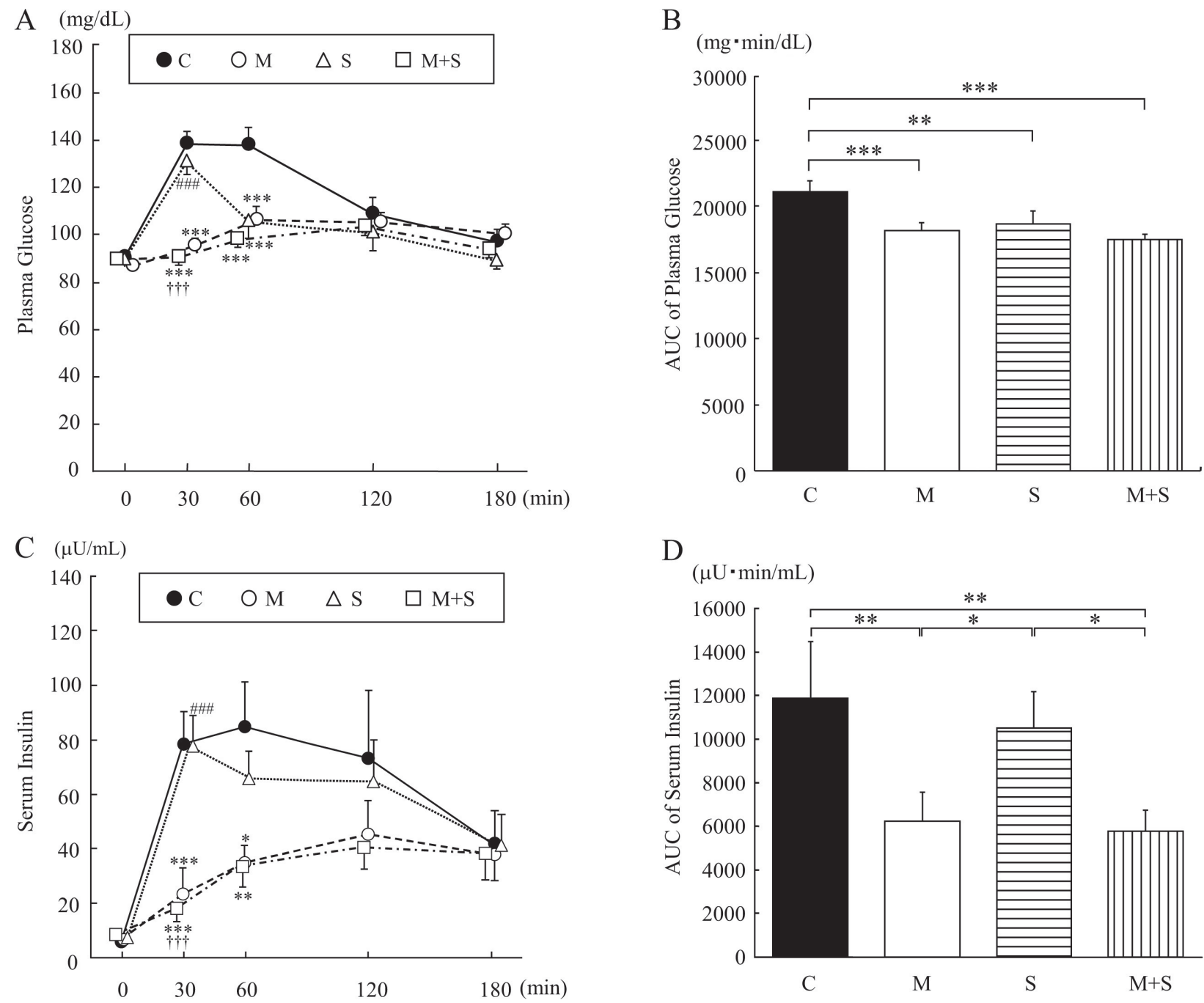

Fig. 1 Area under the curve of the plasma glucose and serum insulin levels for each intake schedule. The time profile and the AUC of the plasma glucose levels are shown in A and B, respectively, while those of the serum insulin levels are shown in C and D, respectively. C: filled circles; M: clear circles; S: triangles; M+S: squares. Number of each group was 10 . Differences with $P$ values of less than 0.05 were considered significant. A and C: ${ }^{*} p<0.05, * * p<0.01, * * * p<0.001 v$ s. C. ${ }^{*} p<0.05,{ }^{*} p<0.01$, ${ }^{\# \# \#} p<0.001$ vs. M. ${ }^{\dagger} p<0.05,{ }^{\dagger \dagger} p<0.01,{ }^{\dagger \dagger} p<0.001$ vs. S. B and D: ${ }^{*} p<0.05,{ }^{* *} p<0.01,{ }^{* * *} p<0.001$ vs. each group.

(Fig. 1A, B), and the AUC of the plasma active GLP-1 level in the $\mathrm{M}+\mathrm{S}$ group was greater than that in the $\mathrm{M}$ and $\mathrm{S}$ groups (Fig. 2B).

Incretins improve hyperglycemia in a glucosedependent fashion, but whether incretins or DPP-4 inhibitors are capable of improving hyperglycemia in individuals with NGT or IGT has remained uncertain. Here, we showed that miglitol and sitagliptin are similarly effective for decreasing postprandial hyperglycemia in non-diabetic men. By contrast, once-daily sitagliptin treatment was reported to have a greater efficacy than thrice-daily voglibose treatment in Japanese patients with type 2 diabetes [16]. Of note, sitagliptin, unlike miglitol, failed to decrease the plasma glucose levels at $30 \mathrm{~min}$ after the start of breakfast (Fig. 1A) but decreased the AUC of the plasma glucose levels (Fig. 1B). This result clearly demonstrates that sitagliptin is effective for improving postprandial hyperglycemia in non-diabetic subjects even though the action of incretin is glucosedependent. Although sitagliptin and vildagliptin were reported to have no effect on postprandial glucose level in subjects with NGT $[13,15]$, we assume that the differences between this study and the previous 
A $(\mathrm{pmol} / \mathrm{mL})$

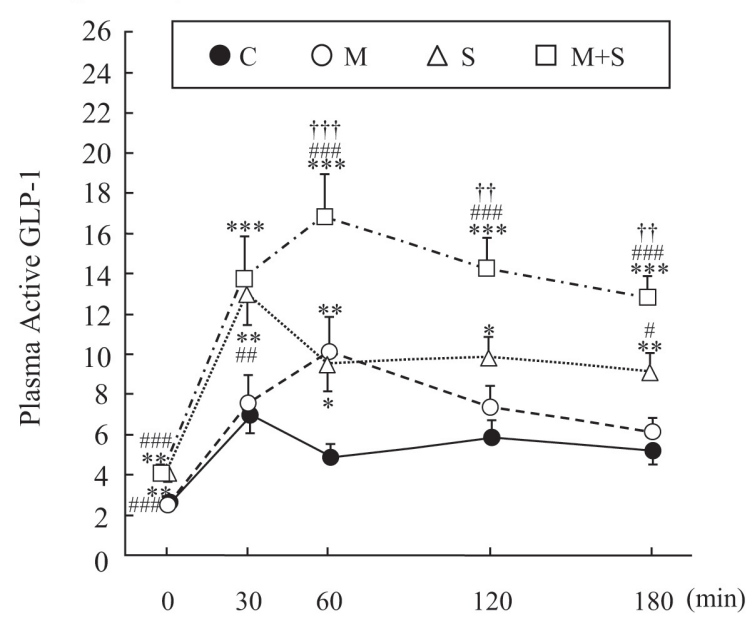

$\mathrm{C}$

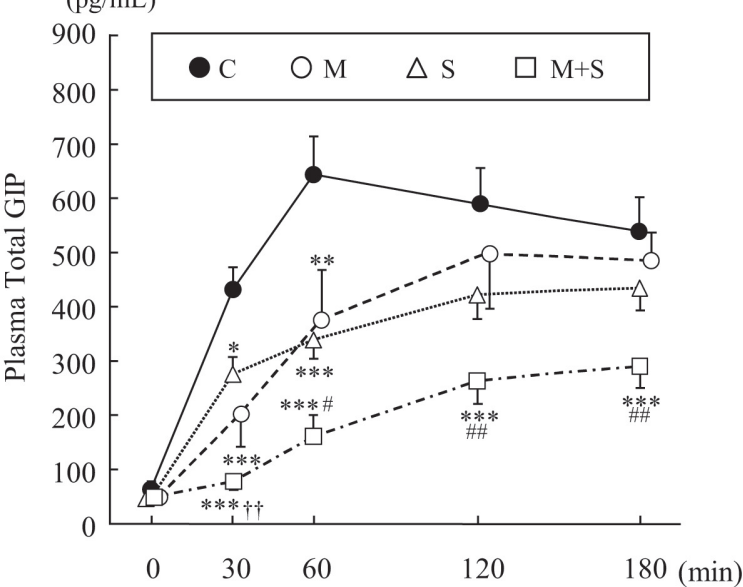

B $(\mathrm{pmol} \cdot \mathrm{min} / \mathrm{mL})$

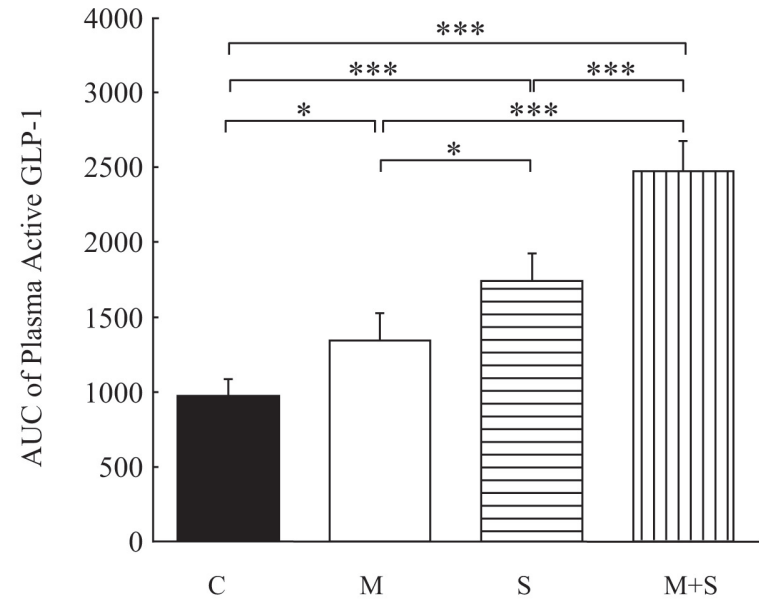

D $\quad(\mathrm{pg} \cdot \mathrm{min} / \mathrm{mL})$

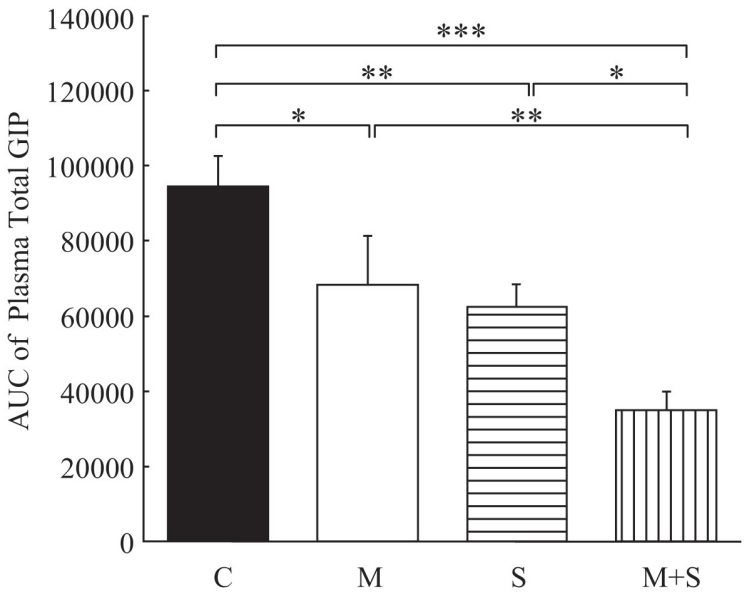

Fig. 2 Area under the curve of the plasma active glucagon-like peptide-1 (GLP-1) and plasma total glucose-dependent insulinotropic polypeptide (GIP) levels for each intake schedule. The time profile and the AUC of the plasma active GLP-1 levels are shown in A and B, respectively, while those of the plasma total GIP levels are shown in C and D, respectively. C: filled circles; M: clear circles; S: triangles; M+S: squares. Number of each group was 10 . Differences with $P$ values of less than 0.05 were considered significant. A and C: ${ }^{*} p<0.05,{ }^{* *} p<0.01, * * * p<0.001 v s$. C. ${ }^{\#} p<0.05,{ }^{\#} p<0.01,{ }^{\# \#} p<0.001 v s$. M. ${ }^{\dagger} p<0.05$, ${ }^{\#} p<0.01,{ }^{\dagger \dagger} p<0.001 v s$. S. B and D: * $p<0.05, * * p<0.01, * * * p<0.001 v s$. each group.

reports may be due to the differences in meals.

Interestingly, sitagliptin decreased the AUC of the blood glucose levels without increasing the insulin levels in non-diabetic subjects (Fig. 1C, D); this effect might be explained by a decrease in the plasma glucagon levels $[8,14]$, although we were unable to measure serum glucagon level in the present study because the antibody against glucagon was not available for commercial use. We would like to measure serum glucagon in future.

The absence of an increase in the serum insulin levels might, in turn, be favorable for sparing insulin secretion by $\beta$ cells in individuals with NGT or IGT.

The active GLP-1 and total GIP profiles in the M group were consistent with those described in previous reports [5-7]. Consistent with other previous reports $[8,14]$, an increase in the AUC of the active GLP-1 levels was observed in the S group. The AUC of active GLP-1 in the $\mathrm{M}+\mathrm{S}$ group was much greater than that in the $\mathrm{M}$ and $\mathrm{S}$ groups. In a recent report, the plasma active GLP-1 levels were higher after treatment with a combination of alogliptin and voglibose, 
compared with after monotherapy with each drug, in diabetic $\mathrm{db} / \mathrm{db}$ mice [17]. Our clinical results in nondiabetic subjects were consistent with this report.

Since active GLP-1 reportedly promotes islet cell growth and inhibits apoptosis in an animal model [9], the increase in active GLP-1 in the $\mathrm{M}$ and S groups and the further increase in the $\mathrm{M}+\mathrm{S}$ group might be beneficial for preserving $\beta$ cells and reducing the development of diabetes in humans. In general, miglitol should be administered before meals; however, the once-daily administration of miglitol as a "GLP-1 enhancer" in combination with sitagliptin might be effective, as demonstrated by the further increase in GLP-1 observed in the M+S group (Fig. 2B). Further study is needed to address this issue.

Interestingly, the AUC of total GIP in the $\mathrm{M}+\mathrm{S}$ group was much smaller than that in the $\mathrm{M}$ and $\mathrm{S}$ groups, and this difference might be due to the combined effect of both drugs. The administration of sitagliptin reportedly increases the active GIP level but decreases the total GIP level [14], consistent with our results. One possible explanation for this phenomenon is that the increased level of active GIP produces a negative feedback inhibition of the total GIP secretion $[8,18]$. AUC of total GIP in the M group was decreased by inhibition of glucose absorption in the upper intestine as reported in [5]. There were no reports which investigate the effects of miglitol on active GIP, however, miglitol is considered to decrease plasma active GIP level due to its pharmacological effect. By contrast, sitagliptin enhanced active GIP levels by inhibiting degradation of the active form rather than increasing secretion, because the ratios of active to total form were increased and total GIP levels were reduced [8]. Inhibition of GIP signaling is beneficial for preventing obesity as reported in [19]. Because administration of miglitol may suppress the increase in active GIP evoked by administration of sitagliptin, combination therapy of these two agents may be useful for obese type 2 diabetic patients.

In conclusion, the results of our study suggest that miglitol, sitagliptin, or their combination contributes to the prevention of the development of type 2 diabetes.

\section{Acknowledgements}

This work was supported in part by a Grant-inAid for Scientific Research (B) 19390251 from the Ministry of Education, Culture, Sports, Science and Technology (MEXT) of Japan, and a Medical Award from the Japan Medical Association.

No potential conflicts of interest relevant to this article exist.

\section{References}

1. Aoki K, Kato H, Terauchi Y(2007) Divided-dose administration of miglitol just before and 15 minutes after the start of a meal smoothes postprandial plasma glucose excursions and serum insulin responses in healthy men. Endocr J 54: 1009-1014.

2. Aoki K, Ito Y, Saito K, Shirakawa J, Togashi Y, Satoh K, Muraoka T, Shinoda K, Masuda K, Kimura M, Terauchi Y(2009) Comparison of pre- versus post-meal administration of voglibose in men with or without impaired glucose tolerance. Diabetes Res Clin Pract 83: e31-32.

3. Chiasson JL, Josse RG, Gomis R, Hanefeld M, Karasik A, Laakso M (2002) STOP-NIDDM Trail Research Group: Acarbose for prevention of type 2 diabetes mellitus: the STOP-NIDDM randomised trial. Lancet 359: 2072-2077.

4. Kawamori R, Tajima N, Iwamoto Y, Kashiwagi A, Shimamoto K, Kaku K (2009) Voglibose Ph-3 Study Group: Voglibose for prevention of type 2 diabetes mellitus: a randomised, double-blind trial in Japanese individuals with impaired glucose tolerance. Lancet 373:1607-1614.

5. Narita T, Katsuura Y, Sato T, Hosoba M, Fujita H, Morii T, Yamada Y (2009) Miglitol induces prolonged and enhanced glucagon-like peptide-1 and reduced gastric inhibitory polypeptide responses after ingestion of a mixed meal in Japanese Type 2 diabetic patients. Diabet Med 26:187-188.

6. Arakawa M, Ebato C, Mita T, Fujitani Y, Shimizu T, Watada H, Kawamori R, Hirose T (2008) Miglitol suppresses the postprandial increase in interleukin 6 and enhances active glucagon-like peptide 1 secretion in viscerally obese subjects. Metabolism 57:1299-1306.

7. Lee A, Patrick P, Wishart J, Horowitz M, Morley JE (2002) The effects of miglitol on glucagon-like peptide- 1 secretion and appetite sensations in obese type 2 diabetics. Diabetes Obes Metab 4:329-335.

8. Herman GA, Bergman A, Stevens C, Kotey P, Yi B, Zhao P, Dietrich B, Golor G, Schrodter A, Keymeulen B, Lasseter KC, Kipnes MS, Snyder K, Hilliard D, 
Tanen M, Cilissen C, De Smet M, de Lepeleire I, Van Dyck K, Wang AQ, Zeng W, Davies MJ, Tanaka W, Holst JJ, Deacon CF, Gottesdiener KM, Wagner JA (2006) Effect of single oral doses of sitagliptin, a dipeptidyl peptidase-4 inhibitor, on incretin and plasma glucose levels after an oral glucose tolerance test in patients with type 2 diabetes. J Clin Endocrinol Metab 91:4612-4619.

9. Farilla L, Hui H, Bertolotto C, Kang E, Bulotta A, Di Mario U, Perfetti R (2002) Glucagon-like peptide-1 promotes islet cell growth and inhibits apoptosis in Zucker diabetic rats. Endocrinology 143: 4397-4408.

10. Terauchi Y, Takamoto I, Kubota N, Matsui J, Suzuki R, Komeda K, Hara A, Toyoda Y, Miwa I, Aizawa $\mathrm{S}$, Tsutsumi S, Tsubamoto Y, Hashimoto S, Eto K, Nakamura A, Noda M, Tobe K, Aburatani H, Nagai R, Kadowaki T (2007) Glucokinase and IRS-2 are required for compensatory beta cell hyperplasia in response to high-fat diet-induced insulin resistance. $J$ Clin Invest 117:246-257.

11. Kadowaki T, Miyake Y, Hagura R, Akanuma Y, Kajinuma H, Kuzuya N, Takaku F, Kosaka K (1984) Risk factors for worsening to diabetes in subjects with impaired glucose tolerance. Diabetologia 26:44-49.

12. Rosenstock J, Foley JE, Rendell M, Landin-Olsson M, Holst JJ, Deacon CF, Rochotte E, Baron MA (2008) Effects of the dipeptidyl peptidase-IV inhibitor vildagliptin on incretin hormones, islet function, and postprandial glycemia in subjects with impaired glucose tolerance. Diabetes Care 31:30-35.

13. Bergman AJ, Stevens C, Zhou Y, Yi B, Laethem M, De Smet M, Snyder K, Hilliard D, Tanaka W, Zeng W, Tanen M, Wang AQ, Chen L, Winchell G, Davies MJ, Ramael S, Wagner JA, Herman GA (2006) Pharmacokinetic and pharmacodynamic properties of multiple oral doses of sitagliptin, a dipeptidyl pepti-
dase-IV inhibitor: a double-blind, randomized, placebo-controlled study in healthy male volunteers. Clin Ther 28:55-72.

14. Bock G, Man CD, Micheletto F, Basu R, Laugen J, Deacon CF, Holst JJ, Toffolo G, Cobelli C, Rizza RA, Vella A (2010) The effect of DPP-4 inhibition with sitagliptin on incretin secretion and on fasting and postprandial glucose turnover in subjects with impaired fasting glucose. Clin Endocrinol (Oxf) 73:189-196.

15. Hu P, Yin Q, Deckert F, Jiang J, Liu D, Kjems L, Dole WP, He YL (2009) Pharmacokinetics and pharmacodynamics of vildagliptin in healthy Chinese volunteers. $J$ Clin Pharmacol 49:39-49.

16. Iwamoto Y, Tajima N, Kadowaki T, Nonaka K, Taniguchi T, Nishii M, Arjona Ferreira JC, Amatruda JM (2010) Efficacy and safety of sitagliptin monotherapy compared with voglibose in Japanese patients with type 2 diabetes: a randomized, double-blind trial. Diabetes Obes Metab 12:613-622.

17. Moritoh Y, Takeuchi K, Hazama M (2010) Combination treatment with alogliptin and voglibose increases active GLP-1 circulation, prevents the development of diabetes and preserves pancreatic beta-cells in prediabetic $\mathrm{db} / \mathrm{db}$ mice. Diabetes Obes Metab12:224-233.

18. Deacon CF, Wamberg S, Bie P, Hughes TE, Holst JJ (2002) Preservation of active incretin hormones by inhibition of dipeptidyl peptidase IV suppresses meal-induced incretin secretion in dogs. $J$ Endocrinol 172:355-362.

19. Miyawaki K, Yamada Y, Ban N, Ihara Y, Tsukiyama K, Zhou H, Fujimoto S, Oku A, Tsuda K, Toyokuni S, Hiai H, Mizunoya W, Fushiki T, Holst JJ, Makino M, Tashita A, Kobara Y, Tsubamoto Y, Jinnouchi T, Jomori T, Seino Y (2002) Inhibition of gastric inhibitory polypeptide signaling prevents obesity. Nat Med 8: 738-742. 